\title{
A Proposal to Reduce Congenital Cerebral Palsy
}

\section{Kazuo Maeda*}

Department of Obstetrics and Gynecology (Honorary Professor), Tottori University Medical School, Yonago, Japan

\begin{abstract}
Aims: To clarify the feasibility to arrest preterm labor to prevent the periventricular leukomalacia (PVL) and Cerebral Palsy (CP) by the study on the developing mechanism of labor contractions.
\end{abstract}

Rational: Fetal periventricular echo density (PVE) precedes preterm neonatal PVL and CP in $18 \%$ of preterm fetuses whose PVE persisted until preterm births. Since no treatment of neonatal PVE appeared immediately after preterm birth was established, another strategy is proposed to prevent preterm neonatal PVE to cease preterm labor until the full term delivery, because no CP developed in full term delivery in our report. Recently, we studied the positive feed-back loop of uterine contraction to the brain by nerves between the uterus and hypothalamus, developing regular labor contractions.

Proposal: The suppression of the nerves of positive feed-back loop by anesthesia or analgesia is our proposal to cease preterm labor until the full term delivery, where no $\mathrm{CP}$ was reported, i.e. the $\mathrm{CP}$, corresponding to $0.2 \%$ of total births, will be reduced.

Conclusion: Regular preterm labor contractions will be ceased by the paralysis of nerves in the positive feed-back loop between the uterus and hypothalamus and the full-term delivery prevents CP, due to disappeared PVE in the neonates born in full term delivery. Clinical feasibility should be investigated in further studies.

Keywords: Fetus; Neonate; PVL; CP; Labor oscillation; Positive feed-back; Uterus and brain innervation; Preterm labor

\section{Introduction}

Periventricular echodensity (PVE) precedes periventricular leukomalacia (PVL) and cerebral palsy (CP) in $18 \%$ of cases, whose PVE persisted through the preterm pregnancy until preterm birth [1] (Figure 1), which corresponds about $0.2 \%$ of all births. There will be two ways to prevent the PVL and CP, i.e. the first is to treat preterm neonate, who develops PVE in the first day of life, to delete the PVE, but no PVE treatment has been established. The second way will be to cease the preterm labor contraction and continue the pregnancy until the full term birth, because no $\mathrm{CP}$ was reported among babies born in full term births [1].

There will be some strategies to prevent the neonatal PVL and CP. The fibrin deposit in the placenta at intervillous space suffers active transfer of glucose and fatty acid from mother to the fetus resulting fetal growth restriction (FGR) where the mother was positive phospholipid antigen and high brightness of placenta with high GLHW tissue characterization, then finally plain oxygen transfer was restricted, resulting in hypoxia, NRFS then fetal death. Fibrinolytic Heparin administration to the mother was effective, fetal estimated weight increased, placental GLHW reduced, and normal neonate was

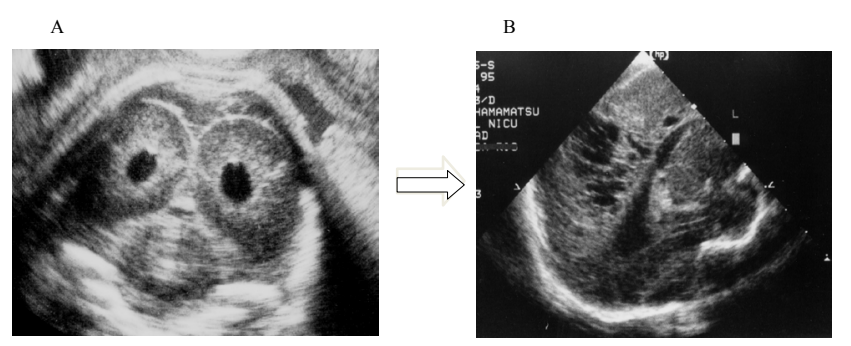

Figure 1: A: A PVE developed in a case of preterm labor persisted until the preterm birth. B: The PVL followed by the CP developed in the preterm neonate of the case A (Courtesy Dr. N.Yamamoto) [6]. obtained by Utsu et al. [2]. Some preterm fetus removed the PVE with high repairing function during pregnancy, maybe due to the increase of growth factor in late pregnancy. Maybe the growth factor disappear in the preterm neonate and the PVE might change to PVL, Such PVE removing factor like as growth factor will be the treatment of postnatal PVE, but unfortunately no treatment has been established yet.

Another treatment will be the suppression of preterm uterine contraction by pharmaceutical tocolysis by beta-adrenergics to prolong the pregnancy to full term birth, because no CP was reported in full term birth [1], but unfortunately the tocolysis was transient and failed to achieve full-term birth. Recently, however, we reported the modern analysis of regular labor uterine contraction. The uterine conntraction cycle was $105 \mathrm{sec}$ determined by intrauterine pressure in 1960 in Uruguay [3], while it was $108 \mathrm{sec}$ by external tocodynamometry in 2000 in Japan [4]. The duration was about $1 \mathrm{~min}$, interval was also 1 min, and the peak intrauterine pressure was 30 to $50 \mathrm{mmHg}$ in normal labor contractions, which was initiated at the pace-maker located at the corner of uterine fundus and conducted by the gap-junction [4] from the fundus to the cervix [3]. However, no mechanism of regular uterine contractions has been clarified. Such regularly repeated waves are seen in electric oscillations. The simulation of the repeated contraction was studied comparing to electric oscillation [5]. Then the treatment of preterm labor will be discussed.

*Corresponding author: Kazuo Maeda, Department of Obstetrics and Gynecology (Honorary Professor), Tottori University Medical School, 3-125, NadamachiYonago, japan, Tel: +81-859-22-6856; Fax: +81-859-22-6856; E-mail: maedak@mocha.ocn.ne.jp

Received October 14, 2013; Accepted October 15, 2013; Published October 19 2013

Citation: Maeda K (2013) A Proposal to Reduce Congenital Cerebral Palsy. J Health Med Informat 4: 135. doi:10.4172/2157-7420.1000135

Copyright: (c) 2013 Maeda K. This is an open-access article distributed under the terms of the Creative Commons Attribution License, which permits unrestricted use, distribution, and reproduction in any medium, provided the original author and source are credited. 


\section{Rationals}

A typical uterine contraction was reported by Caldeyro-Barcia, where repeated uterine contractions were found in amniotic pressure, of which intensity were approx. $40 \mathrm{mmHg}$, duration was approx. one min, and interval also one min [3]. External tocodynamometry was studied comparing to intrauterine pressure changes [4] (Figure 2). The contraction curve was compared to the electronic oscillation in an experimental $2 \mathrm{MHz}$ ultrasound (Figure 3). The regularity of uterine contraction curve resembled the ultrasound waves, however, the difference was the presence of minus deflection of ultrasound waves, which was lost in uterine contraction. This is natural because uterine contraction is a biological uterine muscle action but not the electrical information. Therefore, the uterine contraction curve was conversed upside down to make it minus deflection, then attached to the base of original contraction curve. The combined curve showed a sine wave-like oscillatory change, where it was confirmed that the uterine contraction curve was the part of a biological oscillation (Figure 4). The result made it possible to analyze the labor contraction as an oscillation [5].

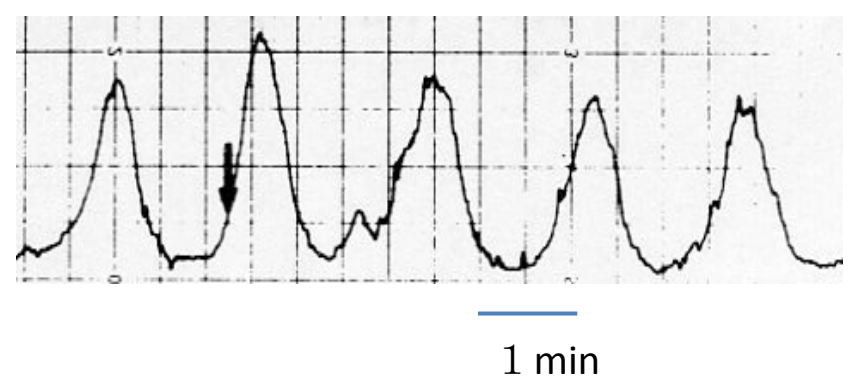

Figure 2: Regular uterine contractions recorded in the first stage of labor. The duration of uterine contraction is about one min and the interval also one $\mathrm{min}$ i.e. the contraction cycle expressed by contraction peak-to-peak time is about two min. The contraction intensity is 30 to $40 \mathrm{mmHg}[3]$.
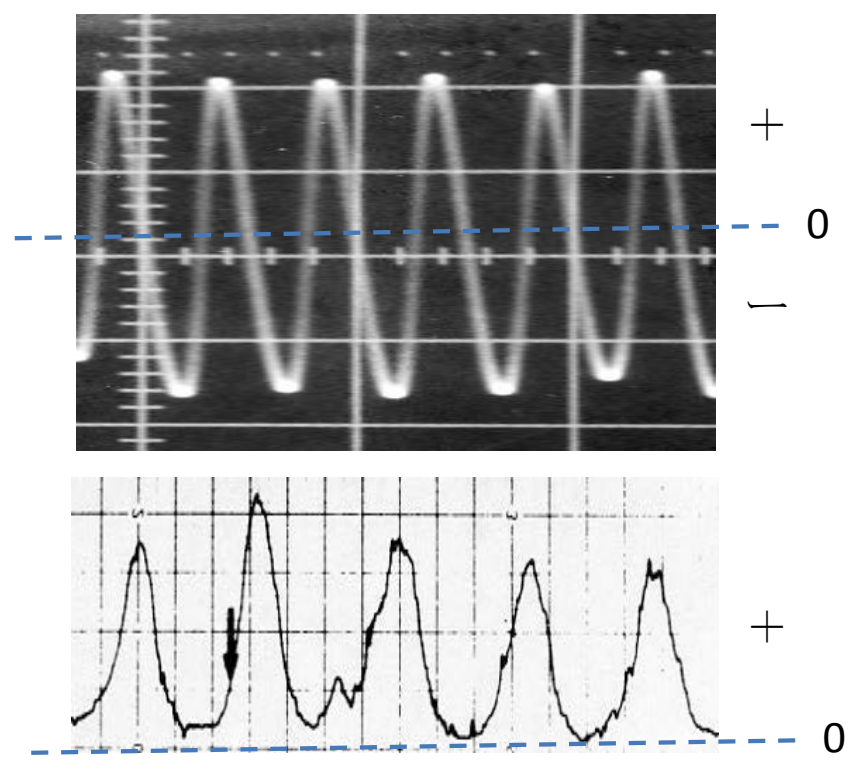

Figure 3: Upper curve is an oscilloscopic image of $2 \mathrm{MHz}$ continuous ultrasound wave,. Lower one is uterine contraction curve illustrated in Figure 1. Both curves resemble each other, but uterine contraction curve has no minus deflection, that is natural because uterine contraction is a biological phenomenon.
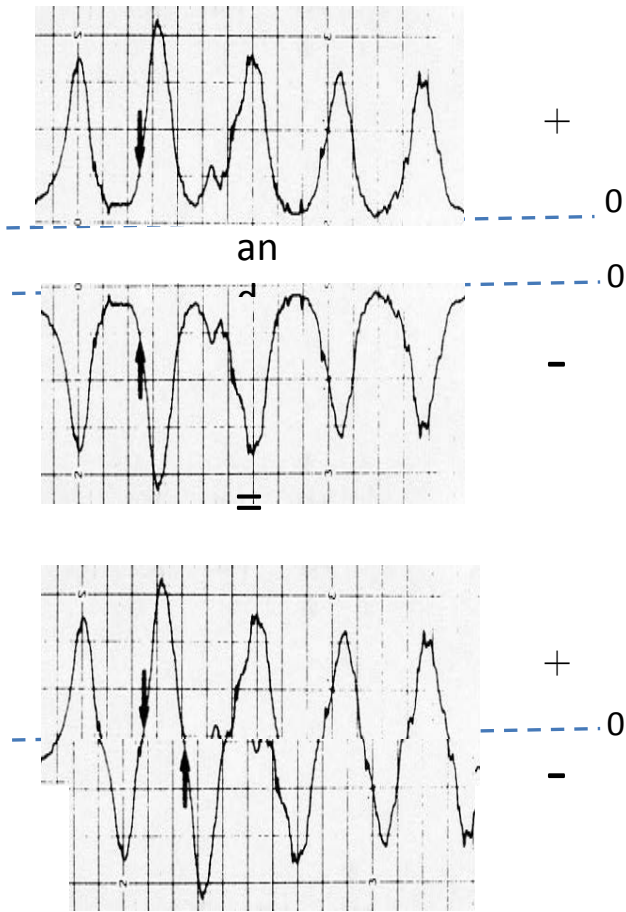

Figure 4: Positive uterine contraction curve (upper line) and reversed virtual negative deflection (middle line) is a sine wave-like oscillation (the lowest pattern), which was able to be analyzed as an oscillation.

Nerves between

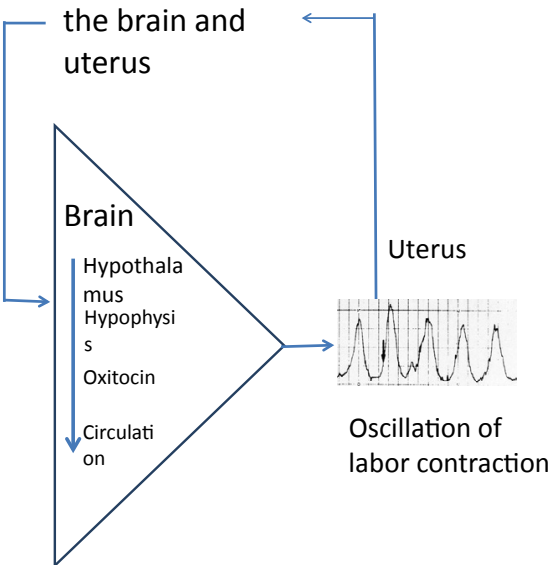

Figure 5: The biological development of oscillatory uterine contractions in normal labor with the biological positive feed-back system including innervations between the uterus and brain [5]

\section{Proposals}

Since a labor contraction of uterus is a biological oscillation, a positive feed-back system is adopted to produce regular oscillation, where the amplifier is composed of the contraction promoting system including hypothalamic center, hypophysis and oxytocin secretion. The contraction amplifying system output is uterine contraction, and the contraction information is fed back to the input of the contraction promoting system through the innervations between the uterus and hypothalamus [6-10] (Figure 5). The frequency of oscillation developed by the biological positive feed-back is approx. $0.008 \mathrm{~Hz}$ (the wave cycle 
is $2 \mathrm{~min}$ in the slow distribution of contraction in the uterus), and the amplitude is approx. $40 \mathrm{mmHg}$. The labor contractions develop regularly with fixed frequency and amplitude in the oscillation, until the delivery of fetus. The most important role of uterus-brain nerves is to maintain the appropriate labor contraction for the delivery of fetus, while the preeclampsia will be caused by the hypothalamic sympathetic center stimulation by enlarged uterus through the nerves [11].

Yamamoto et al. [1] reported the development of PVL followed by the CP in $18 \%$ of fetal PVE cases, when the PVE is preserved in the brain until the preterm delivery. The $\mathrm{CP}$ developmental ratio was about $0.2 \%$ of all births, which was as large as common CP developmental ratio. There could be two CP preventive strategies in the prolonged fetal PVE: the first is to study ultrasound B-mode of all neonate's brain in the day of preterm birth to detect long lasted PVE of the brain, and treat the PVE until its disappearance with any suitable technique, although the treatment has not been established yet.

The second strategy is to totally cease the preterm labor to achieve the birth after 37 weeks of pregnancy, i.e. to deliver the baby in full term births, because no CP was reported by Yamamoto et al in cases of full term births [1]. The pharmacological tocolysis to suppress the preterm labor has been repeatedly tried but failed to achieve full term delivery. Since the preterm labor developed under the positive feedback mechanism, the labor would be ceased by the deep sedation of feedback nerves between the uterus and brain. Analgesia or anesthesia will be effective to make the feed-back system inactive and to cease preterm labor.

\section{Conclusion}

The development of regular labor contraction of uterus is provided by the biological oscillation of positive feed-back system in the loop composed of hypothalamus- hypophysis-oxitocin secretion, uterine contraction and the positive feed-back nerves. Neonatal PVL followed by CP preceded by fetal PVE would be treated by no premature labor by the deep sedation of feed-back nerves between the uterus and brain after further careful studies on new techniques in the future.

\section{References}

1. Yamamoto N, Utsu M, Serizawa M, Ohki S, Maeda K, et al. (2000) Neonatal periventricular leukomalacia preceded by fetal periventricular echo density. Fetal DiagTher 15: 198-208.

2. Utsu M,Maeda K, Kihaile PE (1998) Quantification of sonographic echogenicity with gray-level histogram width: A clinical tissue characterization. Ultrasound Med Biol 24: 225-234.

3. Caldeyro-Barcia R (1960) Factors controlling the actions of the pregnant human uterus. In $\mathrm{M}$ Kowlessar, $5^{\text {th }}$ conference on physiology of prematurity. 1960, Princeton, Josia Macy Foundation, New York, 11-117.

4. Tominaga $Y$, Ito T, Terahara M, Takahasi S, Maeda K (1973) Objective studies on external tocodynamometry. Chuugoku-Shikoku Obstet[Gynecol 24: 25-28.

5. Maeda K (2013) Labor contraction is a biological oscillation of positive feedback system. 36 Ann Conv Japan Soc Mat Fet Med, Miyazaki.

6. Ikeda M, Kawarabayashi T, Sugimori H (1989) Changes in gap junctions in smooth muscle and endometrium of the pegnantrats uterus. Nihon Heikatsukin Gakkai[Zasshi 25: 250-252.

7. Wiesel O,Toth IE, Boldokoi Z, Homyak ABV, Halasz B, et al. (2004) Comparison of transsynaptic viral labeling of central nervous system structures from the uterine horn in virgin, pregnant, and lactating rats. Microsc Res Tech 63: 244 252.

8. Yellon SM,Grushan LA, Rambau GM, Leshuga TJ, Kriby MA (2010) Pregnancyrelated changes in connections from the cervix to forebrain andhypothalamus in mice. Reproduction 140: 155-164.

9. Gnanamanickam GJ, Liewellyn-Smith IJ (2011) Innervation of the rat uterus at esterus: a study in full-thickness, immunoperoxidase-stained whole-mount preparations. J Comp Neurol 519: 621-643.

10. Poletini MO, McKee DT, Szawa RE, Bertram R, Helena CV, et al. (2012) Cervical stimulation activates $\mathrm{A} 1$ and locus coeruleus neurons that project to the paraventricular nucleus of hypothalamus. Brain Res Bull 88: 566-573.

11. Maeda K (2013) Preeclampsia is caused by continuous sympathetic excitation due to an enlarged pregnant uterus. J Perinat Med 12: 1-5. 